\author{
G U E S T E D I T O R I A L
}

\title{
Special Section on Neural Networks and Fuzzy Logic for Imaging Applications
}

\author{
Hong Yan \\ University of Sydney \\ Department of Electrical Engineering \\ New South Wales 2006, Australia \\ Madan M. Gupta \\ University of Saskatchewan \\ College of Engineering \\ Intelligent Systems Research Laboratory \\ Saskatoon, Saskatchewan S7N 5A9, Canada
}

In recent years neural network and fuzzy logic based methods have been used widely in many image processing and pattern recognition applications. Artificial neural networks are developed to mimic the functions of the human brain. Although existing mathematical neural network models are still far less powerful than the biological neurons in terms of their accuracy and flexibility, they are still being used to solve various complicated problems.

Since its inception (Zadek, 1965), fuzzy logic has been an active research topic and has gained many practical applications. For example, fuzzy control and information processing systems have been successfully used in cameras, washing machines, TVs, automobiles, and railway systems. Fuzzy systems are especially useful to deal with noisy, vague, and uncertain data and linguistic variables and rules.

Although neural network models and fuzzy logic systems were developed as two separate fields and seem very different, they share some very important common characteristics. For example, they are both model-free estimators and can generate an arbitrary mapping function between system input and output. The combination of both neural and fuzzy algorithms can often improve system performance and efficiency.

This special section aims to present recent developments of neural and fuzzy algorithms for imaging applications. One paper deals with general image processing problems using neural networks; six papers describe applications of neural and fuzzy algorithms to image thresholding, human face image analysis, character recognition, fingerprint classification, crowding level estimation, and texture identification, respectively; and another paper compares different classifiers for satellite image classification. It is interesting to note that half of the papers make combined use of neural and fuzzy algorithms. Integration of different classification techniques is currently a very active area of further research in pattern recognition. The eight papers selected for this special section are summarized below.

In "Processing of noisy and smalldetailed gray-scale images using cellular neural networks," Aizenberg has described cellular neural network (CNN) structures based on the socalled universal binary neurons (UBNs) and has proposed the network training algorithm. A CNN contains locally connected neurons with feedforward and feedback signals and is suitable for efficient large scale hardware implementation for real-time image processing. The neural network model can be configured to solve many types of problems, such as image filtering and enhancement, noise reduction, and edge detection.

In "Modular expert network approach to histogram thresholding," $\mathrm{Li}$ and Tam have developed a neural network based technique to integrate several image thresholding methods based on histogram analysis. Image thresholding is an important step in many object extraction and recognition systems. One way to find the optimal threshold for an image is to maximize the separability of two classes of pixels based on the pixel gray value distribution, but different criterion functions can produce different threshold values. In $\mathrm{Li}$ and Tam's method, several thresholding procedures are combined using a neural network trained using sample images. The combined thresholding technique is shown to perform better than each individual method.

In "Learning object models from graph templates," Mirhosseini and Yan have developed a method for object detection using a network of neural networks. In their method, each object is represented as a labeled graph and the Gabor features are used as labels of the graph vertices. The Gabor filters resemble the simple cells in the mammalian visual cortex and can be used to represent local image features effectively. Each graph vertex of the object model is learned by a subnetwork and during classification the final decision is made through the majority voting by all subnetworks. The method has been used for detecting the location of eyes in human face images with good result.

In "Recognition of hand-printed Latin characters based on a structural approach with a neural network classifier," Amin, lyer, and Wilson have developed a neural network based method for handwritten character recognition using structural features. The commonly used multilayer perceptron 
network takes a fixed number of numerical inputs and this makes it difficult to deal with qualitative or irregular features. The authors have solved this problem effectively for character recognition. In their method, a character is decomposed into a number of segments and the structural features, such as the type (line, curve, or loop) and position of each segment and the relations between the features, are encoded using binary bit strings, which are then mapped to the input of a neural network.

In "Fingerprint classification using fuzzy cerebellar model arithmetic computer neural networks," Geng and Shen have combined fuzzy representation of input image features and the use of the cerebellar model arithmetic computer (CMAC). The fuzzy functions make the network learning procedure more robust and the network structure simpler. In their system, the KarhunenLoever $(\mathrm{K}-\mathrm{L})$ transform coefficients of fingerprint ridge-flow images are used as input to the fuzzy CMAC network for classification. Good experimental results have been reported for a twoclass problem and the method can be generalized to deal with large scale classification tasks.

In "Estimating the crowding level with a neuro-fuzzy classifier," Boninsegna, Coianiz, and Trentin integrate fuzzy rules with neural networks. In their system, multilayer perceptrons are trained to model individual class membership functions and fuzzy rules are used for classification. Their experiments on estimating the crowding level have shown that the neuro-fuzzy classifier they have used performs substantially better than statistical pattern recognition techniques, such as linear discriminant analysis and nearest neighbor based algorithms.

In "Wavelet based feature-adaptive adaptive resonance theory neural network for texture identification," Wang, Naghdy, and Ogunbona combine fuzzy representation and operations with the adaptive resonance theory (ART) neural network. The fuzzy ART structure has the advantage that it is faster and more reliable for training and simpler to implement than original ART networks. The authors have made use of Gabor wavelets to extract spatial and spatialfrequency features of texture images and have shown that the fuzzy ART network can provide a high classification rate for texture images.

In "Statistical, connectionist, and fuzzy inference techniques for image classification," Israel and Kasabov compare four different classifiers, the parametric maximum likelihood classifier, neural networks, fuzzy rules, and fuzzy neural networks, for classification of satellite images. They have shown that fuzzy neural networks have the overall best performance and can provide additional information, such as rule extraction, and fine tuning of individual classes, which are not available with statistical classifiers.

We would like to thank the Editor of the journal, Professor Edward Dougherty, for his kind support of this special section, the Managing Editor, Ms. Karolyn Labes, for her help with various editorial documents and guidelines, and many reviewers for their valuable time and comments.

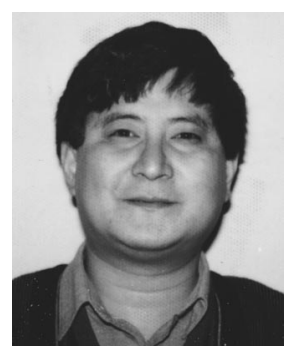

Hong Yan received the $B E$ degree from Nanking Institute of Posts and Telecommunications in 1982 , the MSE degree from the University of Michigan in 1984 and the $\mathrm{PhD}$ degree from Yale University in 1989 , all in electrical engineering. From 1986 to 1989 he was a research scientist at General Network Corporation, New Haven, Connecticut, where he worked on developing a CAD system for optimizing telecommunication systems. Since 1989 he has been with the University of Sydney where he is currently a professor in electrical engineering. His research interests include medical imaging, signal and image processing, neural networks, and pattern recognition. $\mathrm{He}$ is an author or co-author of one book and more than
150 technical papers in these areas. He is a fellow of the Institution of Engineers, Australia (IEAust), a senior member of the IEEE, and a member of SPIE, the International Neural Network Society, the Pattern Recognition Society, and the International Society for Magnetic Resonance in Medicine.

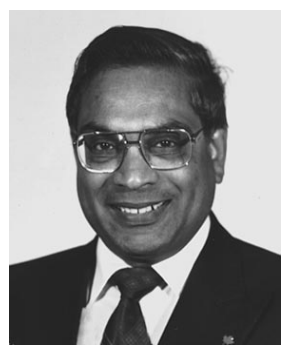

Madan M. Gupta is a Professor of Engineering and the Director of the Intelligent Systems Research Laboratory and the Center of Excellence on NeuroVision Research at the University of Saskatchewan, Saskatoon, Canada. He received the BEng (Honors) in 1961, and the MS in 1962, both in electronics-communications engineering, from the Birla Engineering College (now the BITS), Pilani, India. He received the PhD degree in 1967 from the University of Warwick, United Kingdom. His field of research has been in the areas of adaptive control systems, noninvasive methods for the diagnosis of cardiovascular disease, and fuzzy logic. In addition to publishing more than 550 research papers, he has coauthored two books on fuzzy logic (with Japanese translation), and has edited a set of eighteen books in the field of adaptive control systems and fuzzy logic, fuzzy computing, fuzzy-neural networks, neurovision, and neurocontrol systems. His election to the IEEE fellowship was made for his contributions to the theory of fuzzy sets, adaptive control systems, and advancement of the diagnosis of cardiovascular disease. He was also elected fellow of SPIE for his contributions to the field of neurovision, neurocontrol, and neurofuzzy systems. He has served the engineering community worldwide in various capacities through societies such as IEEE, IFSA, IFAC, SPIE, NAFIPS, UN, and ISUMA. He has been elected as a Visiting Professor and a Special Advisor (in the areas of high technology) to the European Center for Peace and Development (ECPD), University of Peace, established by the United Nations. He is responsible for establishing societies such as the North American Fuzzy Information Processing Society (NAFIPS), and the Canadian Society for Fuzzy Information and Neural Systems (CANS-FINS). 